\title{
Low Dose Imaging Using Simultaneous iDPC- and ADF-STEM for Beam Sensitive Crystalline Structures
}

\author{
Anna Carlsson ${ }^{1}$, Ioannis Alexandrou ${ }^{1}$, Emrah Yücelen ${ }^{1}$, Eric G.T. Bosch ${ }^{1}$, Ivan Lazić ${ }^{1}$ \\ 1. Thermo Fisher Scientific, Materials \& Structural Analysis, Eindhoven, The Netherlands.
}

Imaging of delicate crystal structures such zeolites or metal organic frameworks (MOFs) can be challenging due to their extreme sensitivity to the beam exposure. Therefore it is important that the selected imaging technique has a good signal to noise ratio (SNR), as well as to make use of all of the available electrons during acquisition. As known, conventional STEM techniques such as BF, ABF and (HA)ADF-STEM use only part of the electrons reaching the detector to form the final image. Unlike them, the Integrated Differential Phase Contrast STEM (iDPC-STEM) technique [1, 2], uses the full detector pattern with all of the electrons available. Furthermore, through the integration step, it automatically imposes physical regularization on the noise suppressing the non-conservative parts of the noise field.

iDPC-STEM imaging offers phase contrast information of a thin sample that can be acquired with the same sizes and speeds as regular STEM imaging. That is why simultaneous acquisition with ADFSTEM has become a standard. This enables the user to obtain complementary information on both light and heavy atoms in a thin sample at sub- $\AA$ resolution [3], with extra flexibility for focusing, stigmation and sample manipulation.

Here we present examples of iDPC/ADF-STEM imaging at the limit of contrast and dose. We use the two techniques simultaneously to resolve the atoms at $\AA$ scale resolution for both very light elements and beam sensitive crystalline materials.

The fist example, shown in Fig. 1, involves imaging of lithium, the third element in the periodic system, in a spinel structure $\mathrm{LiTi}_{2} \mathrm{O}_{4}$. We show that $\mathrm{Li}$ can be successfully imaged also in nanoparticles like $\mathrm{LiNi}_{0.5} \mathrm{Mn}_{1.5} \mathrm{O}_{4}$. Using iDPC-STEM we are able to image Li using extremely low electron beam currents as low as a few pA.

The second example, shown in Fig. 2, stresses the huge advantage of using iDPC-STEM imaging at low dose conditions. Zeolites, such as ZSM-5 [010] [4], are examples of very beam sensitive materials unable to withstand more than $\sim 5000 \mathrm{e} / \AA^{2}$. We show high quality atomic resolution imaging using both iDPC-STEM and ADF-STEM at a dose of only $1000 \mathrm{e} / \AA^{2}$. We stress that thanks to this low dose sensitivity of the iDPC-STEM image we are able to focus and stigmate under these low dose conditions and obtain an image that reliably represents the structure down to $1 \AA$ resolution. Using iDPC-STEM we can even monitor gradual sample degradation and find a total dose after which the ZSM-5 structure finally collapses.

[1] I. Lazić, E.G.T. Bosch and S. Lazar, Ultramicroscopy 160 (2016) 265-280.

[2] I. Lazić, E.G.T. Bosch, Advances in Imaging and Electron Physics 199 (2017) 75-184.

[3] E. Yücelen, I. Lazić, E.G.T. Bosch, Scientific Reports 8 (2018) 2676.

[4] J. Su et al, Microporous and Mesoporous Materials 189 (2014) 115-125. 

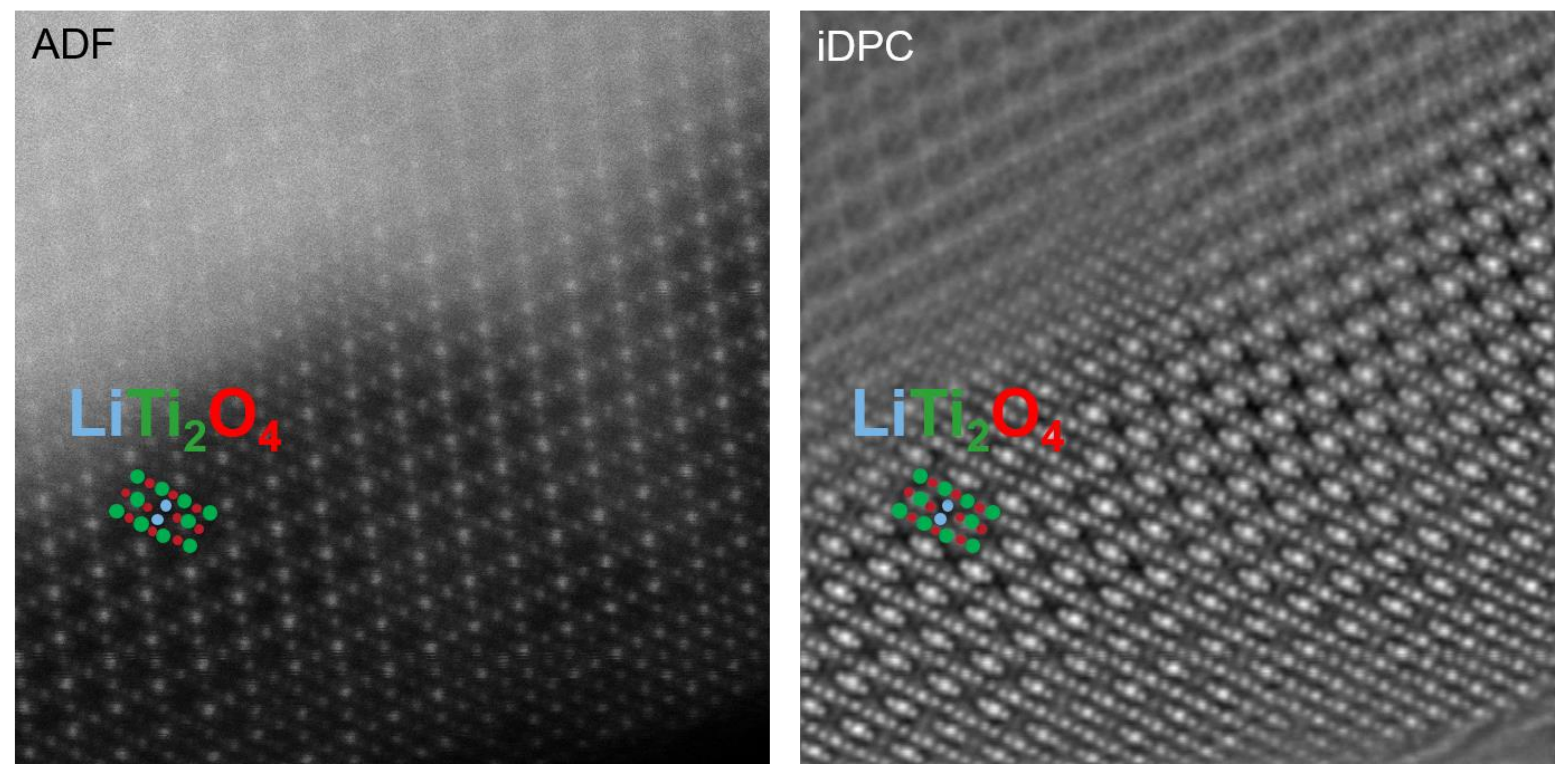

Figure 1. $\mathrm{Li}$ within $\mathrm{LiTi}_{2} \mathrm{O}_{4}$. Simultaneous ADF-STEM (left) with iDPC-STEM (right) acquired at 300 $\mathrm{kV}$ with convergence semi-angle of $20 \mathrm{mrad}$. Both Li and $\mathrm{O}$ columns are visible in iDPC-STEM image.
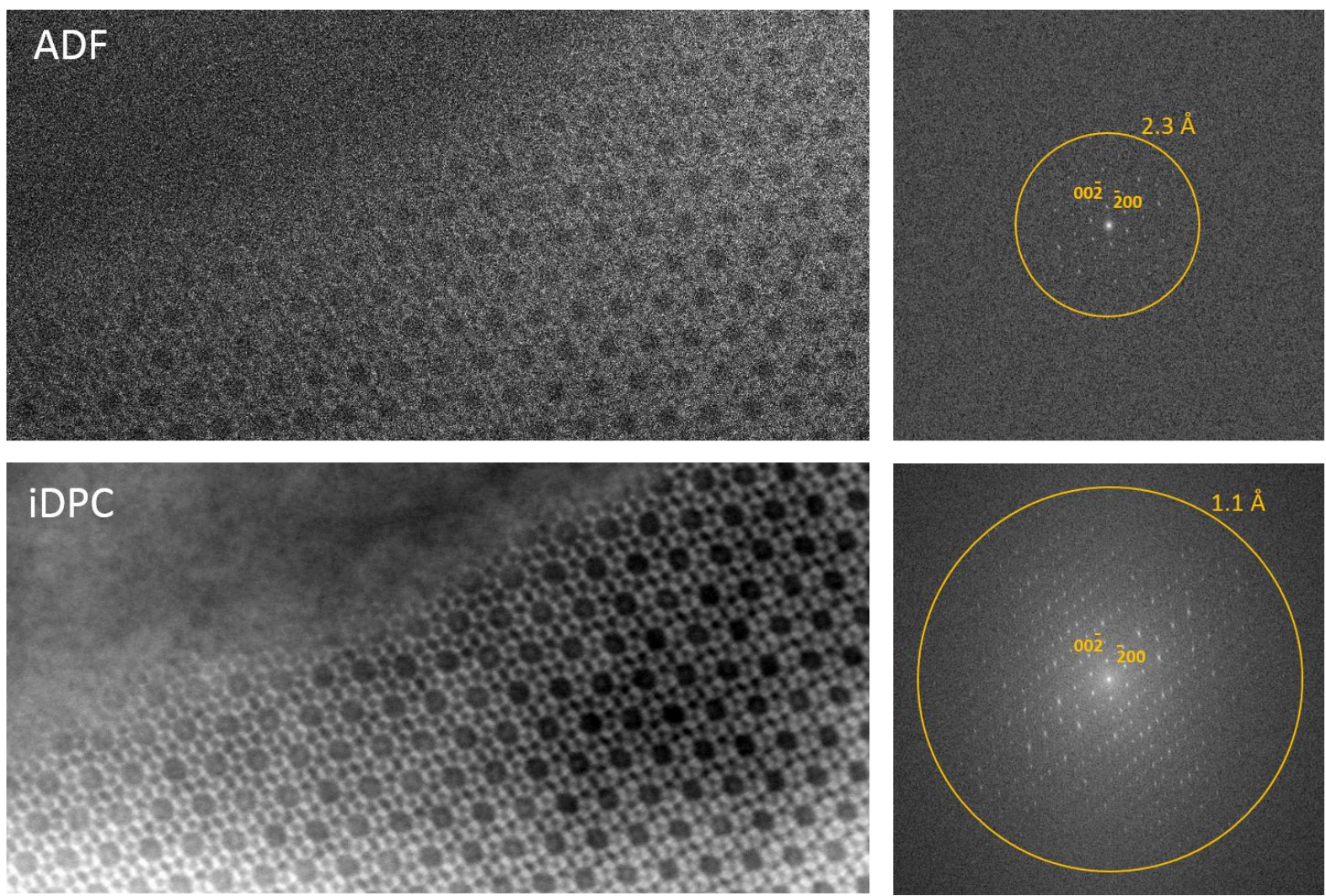

Figure 2. Comparison between ADF-STEM (top) and simultaneous iDPC-STEM (bottom) image (left) of ZSM-5 and their corresponding Fourier transforms (right). Applied dose: $1000 \mathrm{e}^{-} / \AA^{2}$. Images acquired at $300 \mathrm{kV}$ with convergence semi-angle of $20 \mathrm{mrad}$. 